\title{
COMPUTATION OF SINGULARITIES COEFFICIENTS FOR BILAPLACIAN OPERATOR IN A DOMAIN WITH CORNER
}

\section{CHEIKH SECK}

Laboratoire d'Analyse Numérique et d'Informatique (LANI)

BP 234, Université Gaston Berger

Saint-Louis

Sénégal

Département de Mathématiques de la FASTEF, UCAD

Université Cheikh Anta Diop de Dakar

Sénégal

e-mail: cheikh5.seck@ucad.edu.sn

\begin{abstract}
This work consists on the computation of singularities coefficients in a non convex plane domain. We use a mixed finite element method of $C^{0}$ class based on a technical characterisation of dual singular functions. Indeed, these coefficients are obtained directly from dual singular functions. The operator which used here is the bilaplacian involved in the problem $\Delta^{2} u=f$ decomposed in two laplacians $-\Delta u=y$ and $-\Delta y=f$. Throughout the discretization, the singular functions are no longer set up on $u$ but on $y$. In some regularity conditions, the error estimations for the singular coefficients with these hypotheses, give us an error $O\left(h^{2}\right)$ when we use dual singular functions.
\end{abstract}

2010 Mathematics Subject Classification: 58K05, 58K45, 65D20, 65M50, 65M38.

Keywords and phrases: bilaplacian, mixed finite element, singular functions, dual singular functions, error estimations, cracks, simulations, Free-fem++ and Scilab.

Received March 14, 2018; Revised April 3, 2018

(C) 2018 Scientific Advances Publishers 
Numerical tests with Free-fem++ allowed us to calculate and plot the curves of the coefficients of singularities by direct calculations and approximations using finite elements mixed in the same benchmark. Thus, it follows asymptotically that the gap between the two curves is very small narrows and stabilizes when the mesh becomes more refined.

\section{Statement of the Problem}

Let us consider the Dirichlet problem for the bilaplace operator in a domain defined by polar coordinates: $\Omega=\left\{(r, \theta) / r \leq 1,0 \leq \theta \leq \frac{3 \pi}{2}\right\}$, $\Omega \subset \mathbb{R}^{2}$, (Figure 1), bounded open the boundary $\partial \Omega=\Gamma$.

This work deals with the problem described by the following equations:

$$
\left\{\begin{array}{l}
\Delta^{2} u=f \text { in } \Omega, \\
\gamma u=u_{0} \text { on } \Gamma \\
\frac{\partial u}{\partial n}=u_{1} \text { on } \Gamma
\end{array}\right.
$$

where denotes: $\Delta^{2}=\Delta(\Delta)$ and $\frac{\partial u}{\partial n}=\partial_{n}=\nabla u \cdot \vec{n}$ : is the normal derivative on the edge of $\Gamma$. According to the geometry of the open set $\Omega$, the regularity of the solution $u$ does not depend on only the regularity of the data $f, u_{0}$ and $u_{1}$ but also on the geometry of the domain [7]. And, if the problem (1.1) is well posed, it has a singular solution near the summits of nonconvex $\Omega$; and the solution is composed of two parts, one regular and one singular formed from a finite sum of singular functions related to the geometry of the open $\Omega$ [5] and [10]. Indeed, if $f \in H^{-1}(\Omega)$ and the pair $\left(u_{0}, u_{1}\right)$ satisfy minimum conditions of regularities (which are to be specified later on), we know that the solution consists in $u=u_{R}+$ $\sum_{j=1}^{N} \lambda_{j} S_{j}$, where $u_{R} \in H^{3}(\Omega)$ is the regular part and the $\left(S_{i}\right)_{1 \leq i \leq N}$ are the singular functions and $\left(\lambda_{i}\right)_{1 \leq i \leq N}$ are the coefficients of singularity. In 
the case of an open crack, the singularity coefficients associated with the vertex $O$ of the corner are the coefficients of crack propagation. Their effective calculation allows, besides characterizing better the solution $u$, to know a priori the directions and intensities of the crack propagation [9]. Its approximation by finite elements requires consistent discrete spaces of class $C^{1}$. This approach by mixed methods of decomposition $(y=-\Delta u)$ with $-\Delta y=f$ of bilaplacian into problems laplacian of a convergence of order $O\left(h^{2}\right)$. The discretization at hand uses into finite element spaces of type $P 1$. The introduction of singular functions is done on the rotation $y$ and not on the $u$. Firstly, we recall the results on the regularity bilaplacian and the method of dual singular functions in order to write directly the coefficients of singularity without having to calculate $u$ or $y$. Secondly, we obtain the coefficients of singularity as coefficients of the basic functions [4]. We present this unique singular dual method as well as showing improvement to estimate the coefficients of singularities behaviour in $O\left(h^{2}\right)$ for the less regular for all coefficients. Finally, numerical tests are presented and discussed in the last paragraph before the conclusion for this work.

\section{Singularities of Bilaplacian and Dual Functions}

\subsection{Singularities of bilaplacian}

Consider an open bounded, circular, non-convex $\Omega \subset \mathbb{R}^{2}$ with $F$ a corner with the statistical origin $O$ to an orthonormalized reference $(O, I, J)$. Without loss of generality of the study, we assume in the sequel that $\omega>\pi$.

For any point $M$ of $\bar{\Omega}$, we note respectively by $(x, y)$ and $(r, \theta)$ its cartesian and polar coordinates taken from the vertex $O$. Let $r>0, \theta \in[0,2 \pi[$ and $x=r \cos \theta, y=r \sin \theta$. 


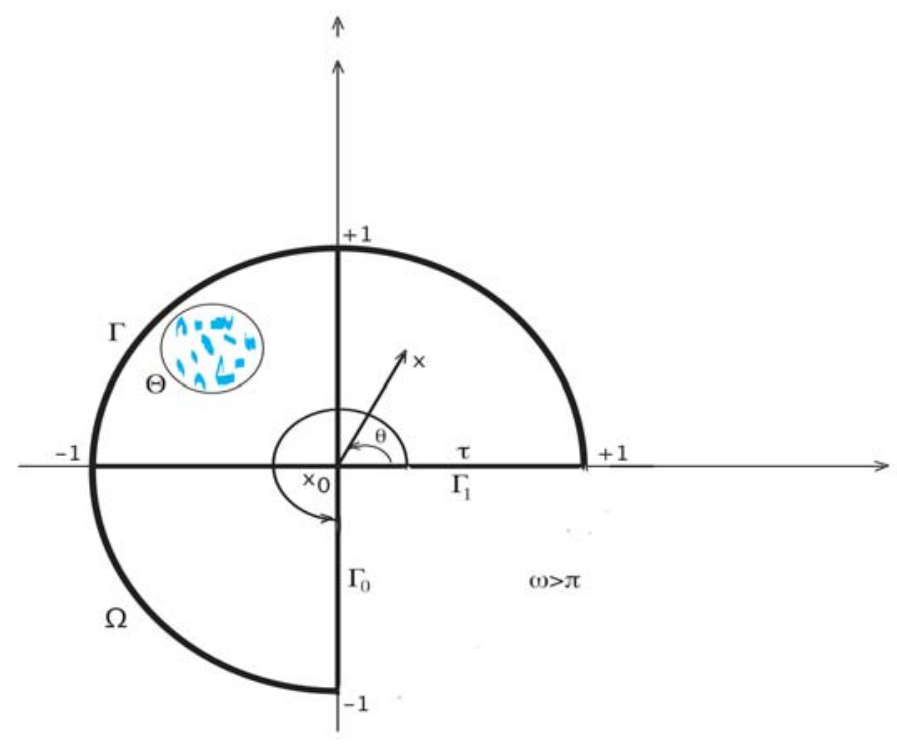

Figure 1. Unit disc with corner.

The boundary $\Gamma$ is characterized by:

$\Gamma_{1}=\{M(r, \theta) \in \partial \Omega ; \theta=0\}=F_{+}, \Gamma_{0}=\left\{M(r, \theta) \in \partial \Omega ; \theta=\frac{3 \pi}{2}\right\}=F_{-}$,

$\Gamma=\bigcup_{i=1}^{N s} \Gamma_{i}, F=F_{+} \cup F_{-}=\Gamma_{0} \cup \Gamma_{1}$ and so $\partial \bar{\Omega}=\bar{\Gamma} \cup \bar{F}$ with $\stackrel{0}{F} \cap \stackrel{0}{\Gamma}=\phi$.

The vertices of the boundary $\partial \Omega$ are defined by: $E_{i+1}=\bar{\Gamma}_{i} \cap \bar{\Gamma}_{i+1}$, $0 \leq i \leq N s$ and $O=\bar{F}_{+} \cap \bar{F}_{-}$. According to the work of [6], we have the following results:

Theorem 2.1. Let $f \in H^{-2}(\Omega)$, $\phi$ and $u \in H^{2}(\Omega)$ such that:

$$
\left\{\begin{array}{l}
\Delta^{2} u=f \text { in } \Omega, \\
u-\phi \in H_{0}^{2}(\Omega) .
\end{array}\right.
$$


(1) If $f \in H^{-1}(\Omega)$, and $\phi \in H^{3}(\Omega)$, the solution $u$ of problem (2.0) decomposes as: $u=u_{R}+\lambda_{1} S_{1}+\lambda_{2} S_{2}$, where $u_{R} \in H^{3}(\Omega), \lambda_{1}, \lambda_{2} \in \mathbb{R}$, with:

$$
\left\{\begin{array}{l}
S_{1}(r, \theta)=r^{\frac{3}{2}}\left(\sin \frac{3}{2} \theta-3 \sin \left(\frac{\theta}{2}\right)\right) \\
S_{2}(r, \theta)=r^{\frac{3}{2}}\left(\cos \frac{3}{2} \theta-\cos \left(\frac{\theta}{2}\right)\right)
\end{array}\right.
$$

and there exists a constant $C(\Omega)>0$, depending only on the domain $\Omega$, such as: $\left\|u_{R}\right\|_{H^{3}(\Omega)}+\sum_{i=1}^{2}\left|\lambda_{i}\right| \leq C(\Omega)\left(\|f\|_{H^{-1}(\Omega)}+\|\phi\|_{H^{3}(\Omega)}\right)$.

(2) If $f \in L^{2}(\Omega)$ and $\phi \in H^{4}(\Omega)$, the solution $u$ decomposes into: $u=u_{R}+\sum_{i=1}^{4} \lambda_{i} S_{i}$, where $u_{R} \in H^{4}(\Omega), \lambda_{1}, \lambda_{2}, \lambda_{3}, \lambda_{4} \in \mathbb{R}$ with:

$$
\left\{\begin{array}{l}
S_{3}(r, \theta)=r^{\frac{5}{2}}\left(\sin \frac{5}{2} \theta-5 \sin \left(\frac{\theta}{2}\right)\right) \\
S_{4}(r, \theta)=r^{\frac{5}{2}}\left(\cos \frac{5}{2} \theta-\cos \left(\frac{\theta}{2}\right)\right)
\end{array}\right.
$$

Remark 2.2. (1) $S_{1}$ and $S_{2}$ being unchanged; there exist a constant $C(\Omega)>0$ depending only of $\Omega$, such that:

$$
\left\|u_{R}\right\|_{H^{4}(\Omega)}+\sum_{i=1}^{4}\left|\lambda_{i}\right| \leq C\left(\|f\|_{L^{2}(\Omega)}+\|\phi\|_{H^{4}(\Omega)}\right) .
$$

(2) The singular functions $\left(S_{i}\right)_{1 \leq i \leq 4}$ are well defined, are biharmonics and $\gamma_{0}\left(S_{i}\right)=\gamma_{1}\left(S_{i}\right)=0$ on $F$, where $\gamma_{0}$ and $\gamma_{1}$ are application traces.

\subsection{Dual singular functions}

We present in this paragraph dual singular functions related to bilaplacian and their properties [6], [10].

Thus, we consider the following functions:

$$
\left\{\begin{array}{l}
T_{1}^{*}(r, \theta)=r^{-1} S_{1}(r, \theta), T_{2}^{*}(r, \theta)=r^{-1} S_{2}(r, \theta), \\
T_{3}^{*}(r, \theta)=r^{-3} S_{3}(r, \theta), T_{4}^{*}(r, \theta)=r^{-3} S_{4}(r, \theta) .
\end{array}\right.
$$


These defined functions satisfy the following properties: for $1 \leq i \leq 4$,

$$
\left\{\begin{array}{c}
\Delta^{2} T_{i}^{*}=0 \text { a.e. in } \Omega, \\
\gamma_{0}\left(T_{i}^{*}\right)=\gamma_{1}\left(T_{i}^{*}\right)=0 \text { on } F .
\end{array}\right.
$$

Thank to [11]:

$$
\begin{aligned}
& \text { - } T_{1}^{*}, T_{2}^{*} \in H^{s+1}(\Omega) \text { and } T_{3}^{*}, T_{4}^{*} \in H^{s}(\Omega), \forall s \in\left[0, \frac{1}{2}[.\right. \\
& \text { - } T_{1}^{*}, T_{2}^{*} \in W^{2, s}(\Omega) \text { and } T_{3}^{*}, T_{4}^{*} \in W^{1, s}(\Omega), \forall s \in\left[1, \frac{4}{3}[.\right.
\end{aligned}
$$

Let $\mathcal{B}$ be an open nonempty party of $\Omega$ such that the origin $O \notin \partial \mathcal{B}$, we have $\left.T_{i}^{*}\right|_{\overline{\mathcal{B}}} \in C^{\infty}(\mathcal{B})$ for $1 \leq i \leq 4$.

We define the dual singular functions $\left(S_{i}^{*}\right)_{1 \leq i \leq 4}$ by posing: $S_{i}^{*}=T_{i}^{*}-\phi_{i}^{*}$ for $1 \leq i \leq 4$ with the functions $\phi_{i}^{*}$ solutions of the Equation (2.4).

\subsection{Characterization of singularity coefficients}

By using the dual singular functions, we describe direct calculation of coefficients of singularity from the four dual singular functions $\left(S_{i}^{*}\right)_{1 \leq i \leq 4}$, data $f$ and $\phi$ solution of problems (1.1) and (2.0). Note $\rho^{*}=d(O, \Gamma)$ and consider all truncation functions defined as follows: $\tau=\left\{\chi \in C^{\infty}\left(\mathbb{R}^{+}, \mathbb{R}^{+}\right) / \exists r_{0}, r_{1} \in \mathbb{R}\right.$ fixed, $\left.0<r_{0}<r_{1}<\rho^{*}\right\}$, where

$$
\chi(\Omega)=\left\{\begin{array}{c}
1 \text { if } 0 \leq r \leq r_{0}, \\
0, \text { if } r_{1} \leq r .
\end{array}\right.
$$

Also, we define, for any $\rho \in] 0, \rho^{*}[$, the coefficients:

$$
k_{i j}(\rho)=\int_{\{r=\rho\}}\left(\Delta T_{i}^{*} \partial_{r} S_{j}-S_{j} \partial_{r} \Delta T_{i}^{*}+T_{i}^{*} \partial_{r} \Delta S_{j}-\Delta S_{j} \partial_{r} T_{i}^{*}\right) r d \theta \text { for } 1 \leq i, j \leq 4 .
$$


Also according to [2], we have

$$
\left\{\begin{array}{l}
k_{i j}=a_{i} \pi \delta_{i j} \text { with } \\
a_{1}=8, a_{2}=a_{3}=24 \text { and } a_{4}=-120 .
\end{array}\right.
$$

\subsection{Discretization of the domain}

The finite element method that we are going to address allows to reach high levels of convergence when the domain can be triangulated, which obviously implies that the open $\Omega$ is polygonal in $\mathbb{R}^{2}$. We will consider our domain $\Omega$ bounded in $\mathbb{R}^{2}$ and the boundary regular pieces. It appears natural to us to begin by approaching the open $\Omega$ by the unit disk with corner into a polygonal open $\Omega_{h}$, the vertices of the boundary $\Gamma_{h}$ of $\Omega_{h}$ are the points $E_{i+1}^{h}=\bar{\Gamma}_{i}^{h} \cap \bar{\Gamma}_{i+1}^{h}$, with $0 \leq i \leq N s$ of the boundary $\Gamma_{h}$ of the domain. Let $\mathbb{T}_{h}$ a regular decomposition of $\bar{\Omega}$ into triangles (triangulation) $K$. We notice, for each triangle $K, h_{K}$ its diameter and mesK its area. Let's $h=\max _{K \in \mathbb{T}_{h}} h_{K}$ and assume that exists a constant $C>0$ independent of $h$, such that

$$
C h_{K}^{2} \leq \operatorname{mesK} \quad \forall K \in \mathbb{T}_{h} .
$$

Consider the following subspace of $H^{1}(\Omega)$ defined by: $X=\left\{\theta \in H^{1}(\Omega) /\right.$ $\left.\forall K \in \mathbb{T}_{h}, \theta_{\mid K} \in H^{2}(K)\right\}$ and one takes the discrete spaces following: $X_{h}=\left\{v_{h} \in C^{0}(\bar{\Omega}) / v_{\left.h\right|_{P_{1}}} \in P_{1}(K), \forall K \in \mathbb{T}_{h}\right\} \quad$ and $\quad M_{h}=X_{h} \cap H_{0}^{1}(\Omega)$, where $P_{1}(K)$ is the affine space of polynomial functions on $K$. Also, we note: $y_{S_{i}}=-\Delta S_{i}$, for $1 \leq i \leq 4$ and we can deduce that

$$
\Delta y_{S_{i}}=-\Delta^{2} S_{i}=0, \quad \forall 1 \leq i \leq 4
$$


Moreover, consider the discrete space with the addition of singularities $H_{h}^{N}, N \in\{2,4\}$ defined

$$
H_{h}^{N}= \begin{cases}X_{h} \oplus_{i=1}^{2}\left\{y_{S_{i}}\right\} & \text { for } N=2, \\ X_{h} \oplus_{i=1}^{4}\left\{y_{S_{i}}\right\} & \text { for } N=4\end{cases}
$$

\section{Mixed Variational Formulation}

In the sequel, we introduce the following functional spaces: $W=\left\{\theta \in H^{3}(\Omega) / \gamma_{0}(\theta)=\gamma_{1}(\theta)=0\right.$ a.e. on $\left.F\right\}$ and $H^{-1}(\Delta, \Omega)=\left\{\theta \in L^{2}(\Omega)\right.$, $\left.\Delta \theta \in H^{-1}(\Omega)\right\}$ with the natural norm following:

$$
\forall \theta \in H^{-1}(\Delta, \Omega), \quad\|\theta\|_{H^{-1}(\Delta, \Omega)}^{2}=\|\theta\|_{L^{2}(\Omega)}^{2}+\|\Delta \theta\|_{H^{-1}(\Omega)}^{2} .
$$

Note that also:

- $H^{-1}(\Delta, \Omega)$ is a Hilbert space.

- $D(\bar{\Omega})$ is dense in $H^{-1}(\Delta, \Omega)$.

- $H^{1}(\Omega) \subset H^{-1}(\Delta, \Omega)$.

We define the bilinear form $a(.$, .) :

$$
\forall \theta, \delta \in L^{2}(\Omega), a(\theta, \delta)=\int_{\Omega} \theta \delta d \Omega
$$

and the linear form $b(.,$.$) :$

$\forall \theta \in H^{-1}(\Delta, \Omega), \forall v \in H_{0}^{1}(\Omega), b(\theta, v)=<\Delta \theta, v{ }_{H^{-1}(\Omega), H_{0}^{1}(\Omega)}=-\int_{\Omega} \nabla \theta \nabla v d \Omega$.

We also define the discrete bilinear form:

$$
\forall \theta, \delta \in X, a_{h}(\theta, \delta)=\sum_{K \in \mathbb{T}} m e s \mathbb{T} \int_{\mathbb{T}} \partial_{n} \theta \partial_{n} \delta d \mathbb{T} .
$$




\section{Main Results}

This is to estimate errors on the coefficients of singularities by a method using both the singular functions and singular dual functions. Coefficients of singularities $\lambda_{i}$ are characterized by the unique dual functions $S_{i}^{*}$ in $\Omega$ and $\partial_{n} \rho_{i}^{*}$ on the edge. The coefficients of discrete singularities $k_{h_{i}}$ are defined naturally by the dual method.

Theorem 4.1. (1) If $f \in H^{-1}(\Omega)$ and $\psi \in H^{3}(\Omega) \cap W$ and $i \in\{1,2\}$, then we have:

$$
\pi a_{i} k_{h_{i}}=-<f, S_{h_{i}}^{*}>_{H^{-1}(\Omega), H_{0}^{1}(\Omega)}+\int_{\Gamma} \gamma_{1}(\psi) \partial_{n} \rho_{h_{i}}^{*} d \Gamma-\int_{\Gamma} \gamma_{0}(\psi) \partial_{n} \rho_{h_{i}}^{*} d \Gamma
$$

(2) If $f \in L^{2}(\Omega)$ and $\psi \in H^{4}(\Omega) \cap W$ and $i \in\{3,4\}$, then we have:

$$
\pi a_{i} k_{h_{i}}=-\int_{\Omega} f S_{h_{i}}^{*} d \Omega+\int_{\Gamma} \gamma_{1}(\psi) \partial_{n} \rho_{h_{i}}^{*} d \Gamma-\int_{\Gamma} \gamma_{0}(\psi) \partial_{n} \rho_{h_{i}}^{*} d \Gamma
$$

For proof of this Theorem 4.1, we need the following fundamental lemma:

Lemma 4.2. (1) If $f \in H^{-1}(\Omega)$ and $\psi \in W$, then we have:

$$
\begin{aligned}
\pi a_{i} k_{i}= & -<f, S_{i}^{*}{ }_{H^{-1}(\Omega), H_{0}^{1}(\Omega)}-\int_{\Gamma} \gamma_{0}(\psi) \gamma_{1}\left(\Delta S_{i}^{*}\right) d \Gamma \\
& +\int_{\Gamma} \gamma_{1}(\psi) \gamma_{0}\left(\Delta S_{i}^{*}\right) d \Gamma, \text { for } 1 \leq i \leq 2 .
\end{aligned}
$$

(2) If $f \in L^{2}(\Omega)$ and $\psi \in H^{4}(\Omega) \cap W$, then we have:

$$
\pi a_{i} k_{i}=-\int_{\Omega} f S_{i}^{*} d \Omega-\int_{\Gamma} \gamma_{0}(\psi) \gamma_{1}\left(\Delta S_{i}^{*}\right) d \Gamma+\int_{\Gamma} \gamma_{1}(\psi) \gamma_{0}\left(\Delta S_{i}^{*}\right) d \Gamma, \text { for } 1 \leq i \leq 4
$$

where $S_{i}^{*}$ : dual singular functions. 


\section{Proof of Lemma 4.2.}

(1) If $f \in H^{-1}(\Omega)$ and $\psi \in W$, we have for $i=1,2$ and $\forall \chi \in \tau$

$$
\begin{aligned}
& <f, S_{i}^{*}{ }_{H^{-1}(\Omega), H_{0}^{1}(\Omega)}=<\Delta^{2} \psi^{r}, S_{i}^{*}{ }^{-1}(\Omega), H_{0}^{1}(\Omega) \\
& =<\Delta^{2} \psi^{r},(1-\chi) S_{i}^{*}>_{H^{-1}(\Omega), H_{0}^{1}(\Omega)}+<\Delta^{2} \psi^{r}, \chi S_{i}^{*}>_{H^{-1}(\Omega), H_{0}^{1}(\Omega)} .
\end{aligned}
$$

We know that for all $\chi \in \tau$ and integrating by parts:

$$
\begin{aligned}
& <\Delta^{2} \psi, \chi S_{i}^{*}{ }_{H^{-1}(\Omega), H_{0}^{1}(\Omega)}=\int_{\Omega} \psi \Delta^{2}\left(\chi S_{i}^{*}\right) d \Omega, \quad i=1,2, \forall \psi \in W, \\
& \int_{\Omega} \chi S_{i}^{*} \Delta^{2} \psi d \Omega=\int_{\Omega} \psi \Delta^{2}\left(\chi S_{i}^{*}\right) d \Omega, \quad i=3,4, \forall \psi \in W \cap H^{4}(\Omega) .
\end{aligned}
$$

Thus

$$
<f, S_{i}^{*}{ }_{H^{-1}(\Omega), H_{0}^{1}(\Omega)}=\int_{\Omega} \Delta \psi^{r} \Delta\left[(1-\chi) S_{i}^{*}\right] d \Omega+\int_{\Omega} \psi^{r} \Delta^{2}\left(\chi S_{i}^{*}\right) d \Omega .
$$

Also $\psi=\psi^{r}+\sum_{j=1}^{2} \lambda_{j} S_{j}$ and

$$
\begin{gathered}
\sum_{j=1}^{2} \lambda_{j} k_{i j}=\pi \lambda_{i} a_{i} \\
=\sum_{j=1}^{2} \lambda_{j}\left(\int_{\Omega} \Delta S_{j} \Delta(1-\chi) S_{i}^{*} d \Omega+\int_{\Omega} S_{j} \Delta^{2}\left(\chi S_{i}^{*}\right) d \Omega\right) .
\end{gathered}
$$

By adding member to member relations (4.5) and (4.7), we get:

$$
\begin{aligned}
<f, S_{i}^{*}{ }_{H^{-1}(\Omega), H_{0}^{1}(\Omega)} & +\sum_{j=1}^{2} \lambda_{j} k_{i j}=\int_{\Omega} \Delta \psi^{r} \Delta\left[(1-\chi) S_{i}^{*}\right] d \Omega+\int_{\Omega} \phi^{r} \Delta^{2}\left(\chi S_{i}^{*}\right) d \Omega \\
& +\sum_{j=1}^{2} \lambda_{j}\left(\int_{\Omega} \Delta S_{j} \Delta(1-\chi) S_{i}^{*} d \Omega+\int_{\Omega} S_{j} \Delta^{2}\left(\chi S_{i}^{*}\right) d \Omega\right)
\end{aligned}
$$




$$
\begin{aligned}
& =\int_{\Omega}\left(\Delta \psi^{r}+\sum_{j=1}^{2} \lambda_{j} \Delta S_{j}\right) \Delta\left[(1-\chi) S_{i}^{*}\right] d \Omega+\int_{\Omega}\left(\psi^{r}+\sum_{j=1}^{2} \lambda_{j} S_{j}\right) \Delta^{2}\left(\chi S_{i}^{*}\right) d \Omega \\
& =\int_{\Omega} \Delta \psi \Delta\left[(1-\chi) S_{i}^{*}\right]+\psi \Delta^{2}\left(\chi S_{i}^{*}\right) d \Omega .
\end{aligned}
$$

Furthermore, we know that: $-\Delta^{2}\left(\chi S_{i}^{*}\right)=\Delta^{2}\left[(1-\chi) S_{i}^{*}\right]$.

Thus we have:

$$
\begin{array}{r}
\int_{\Omega} \Delta \psi \Delta\left[(1-\chi) S_{i}^{*}\right]+\int_{\Omega} \psi \Delta^{2}\left(\chi S_{i}^{*}\right) d \Omega=\int_{\Omega} \Delta \psi \Delta\left[(1-\chi) S_{i}^{*}\right] d \Omega-\int_{\Omega} \psi \Delta^{2}(1-\chi) S_{i}^{*} d \Omega \\
=\left(\int_{\partial \Omega} \gamma_{1}(\psi) \gamma_{0}\left(\Delta(1-\chi) S_{i}^{*}\right) d \Gamma-\int_{\partial \Omega} \gamma_{0}(\psi) \gamma_{1}\left(\Delta(1-\chi) S_{i}^{*}\right) d \Gamma\right)
\end{array}
$$

(2) If $f \in L^{2}(\Omega)$ and $\psi \in H^{4}(\Omega) \cap W$, we have for all $\chi \in \tau$

$$
\begin{aligned}
\int_{\Omega} f S_{i}^{*} d \Omega & =\int_{\Omega} S_{i}^{*} \Delta^{2} \psi^{r} d \Omega \\
& =\int_{\Omega}(1-\chi) S_{i}^{*} \Delta^{2} \psi^{r} d \Omega+\int_{\Omega} \chi S_{i}^{*} \psi \Delta^{2} \psi^{r} d \Omega \\
& =\int_{\Omega} \Delta \psi^{r} \Delta\left[(1-\chi) S_{i}^{*}\right] d \Omega+\int_{\Omega} \psi^{r} \Delta^{2}\left(\chi S_{i}^{*}\right) d \Omega .
\end{aligned}
$$

In the same way as (1): $\psi=\psi^{r}+\sum_{j=1}^{4} \lambda_{j} S_{j}$ and

$$
\begin{gathered}
\sum_{j=1}^{4} \lambda_{j} k_{i j}=\pi \lambda_{i} a_{i} \\
=\sum_{j=1}^{4} \lambda_{j}\left(\int_{\Omega} \Delta S_{j} \Delta(1-\chi) S_{i}^{*} d \Omega+\int_{\Omega} S_{j} \Delta^{2}\left(\chi S_{i}^{*}\right) d \Omega\right) .
\end{gathered}
$$


As in (1) by adding member to member in (4.8) and (4.12), we have

$$
\begin{aligned}
\int_{\Omega} f S_{i}^{*} d \Omega+\sum_{j=1}^{4} \lambda_{j} k_{i j} & =\int_{\Omega} f S_{i}^{*} d \Omega+\pi \lambda_{i} a_{i} \\
& =\int_{\Omega} \Delta \psi \Delta\left[(1-\chi) S_{i}^{*}\right]+\psi \Delta^{2}\left(\chi S_{i}^{*}\right) d \Omega
\end{aligned}
$$

Hence, considering the decomposition in singular and regular part, we deduce the result as in (1).

Remark 4.3. This lemma allowed us to determine the coefficients of singularities without having to calculate the approximate solution of the problem (1.1), all we need is an approximation of singular solutions dual $\left(S_{i}^{*}\right)_{1 \leq i \leq 4}$ in a space of elements mixed finite.

\section{Proof of Theorem 4.1.}

We will approach $S_{i}^{*}$ in $\Omega$ and $\Delta S_{i}^{*}$ on $\Omega$, respectively by $S_{h_{i}}^{*}$ and $\Delta S_{h_{i}}^{*}$ in the corresponding discrete spaces. Let us pose: $S_{i}^{*}=T_{i}^{*}-\phi_{i}^{*}$, $1 \leq i \leq 4$.

Let $\chi$ a truncation function, and note: $\psi_{i}^{*}=-(1-\chi) T_{i}^{*}+\phi_{i}^{*} \in H_{0}^{2}(\Omega)$.

Thus we obtain: $S_{i}^{*}=\chi T_{i}^{*}-\psi_{i}^{*}$ and let $\rho_{i}^{*}=-\Delta \phi_{i}^{*} \in L^{2}(\Omega)$.

Let $\quad \mathcal{F}_{i}=\Delta^{2}\left(\chi T_{i}^{*}\right) \in L^{2}(\Omega)$, with $\left\|\mathcal{F}_{i}\right\|_{L^{2}(\Omega)} \leq C$, where $C$ is a constant independent of $\chi$, we deduce that: $\Delta^{2} \psi_{i}^{*}=\mathcal{F}_{i}$ a.e. in $\Omega$.

And, we can see when $\Delta S_{i}^{*}=\Delta\left(\chi T_{i}^{*}\right)-\Delta \psi_{i}^{*}=\rho_{i}^{*}$ on the open $\Omega$.

Therefore, $\partial_{n} \Delta S_{i}^{*}=\partial_{n} \Delta\left(\chi T_{i}^{*}\right)-\partial_{n} \Delta \psi_{i}^{*}=\partial_{n} \rho_{i}^{*}$ on the boundary $\Gamma$ hence $\lambda_{i}$ is characterized by $S_{i}^{*}$ in the domain $\Omega$ and by $\partial_{n} \rho_{i}^{*}$ on the boundary $\Gamma$. 
Now consider the following discrete variational problem:

$$
\left\{\begin{array}{c}
a_{h}\left(\rho_{h_{i}}^{*}, \theta_{h}\right)+b\left(\theta_{h}, \theta_{h_{i}}^{*}\right)=0, \quad \forall \theta_{h} \in H_{h}^{N}, \\
b\left(\rho_{h_{i}}^{*}, \eta_{h}\right)=\int_{\Omega} \eta_{h} \mathcal{F}_{i} d \Omega, \quad \forall \eta_{h} \in M_{h}, \\
\rho_{h_{i}}^{*} \in H_{h}^{N}, \phi_{h_{i}} \in M_{h} .
\end{array}\right.
$$

The variational problem (4.15), has a unique solution in the space discretized $H_{h}^{N}$ from the mixed variational formulation of the above. We remark that $\rho_{h_{i}}^{*}$ approach $\rho_{i}^{*}$ and $\phi_{h_{i}}^{*}$ approach $\phi_{i}^{*}$. Similarly we define the approximation $S_{h_{i}}^{*}$ of $S_{i}^{*}$ by the relation $S_{h_{i}}^{*}=\chi T_{h_{i}}^{*}-\psi_{h_{i}}^{*}$ for $1 \leq i \leq 4$ and coefficients of singularities are naturally defined by the dual method. Therefore by Lemma 4.2, we obtain:

(i) If $f \in H^{-1}(\Omega)$ and $\psi \in H^{3}(\Omega) \cap W$ and $i \in\{1,2\}$

$$
\pi a_{i} k_{h_{i}}=-<f, S_{h_{i}}^{*}>_{H^{-1}(\Omega), H_{0}^{1}(\Omega)}+\int_{\Gamma} \gamma_{1}(\psi) \partial_{n} \rho_{h_{i}}^{*} d \Gamma-\int_{\Gamma} \gamma_{0}(\psi) \partial_{n} \rho_{h_{i}}^{*} d \Gamma .
$$

(ii) If $f \in L^{2}(\Omega)$ and $\psi \in H^{4}(\Omega) \cap W$ and $i \in\{3,4\}$

$$
\pi a_{i} k_{h_{i}}=-\int_{\Omega} f S_{h_{i}}^{*} d \Omega+\int_{\Gamma} \gamma_{1}(\psi) \rho_{h_{i}}^{*} d \Gamma-\int_{\Gamma} \gamma_{0}(\psi) \partial_{n} \rho_{h_{i}}^{*} d \Gamma
$$

\section{Numerical Tests}

The numerical tests below were conducted on the unit disc with corner Figure 1 with the software Free-fem++ version 3.17 of University Pierre and Marie Curie Laboratory J.L LIONS and INRIA. The numerical results have been tested on uniform mesh whose characteristics are described in the table below. 
Table 1. Characteristics of the meshes tested

\begin{tabular}{|c|c|c|c|c|c|c|c|c|}
\hline Parameters $N$ & 100 & 200 & 300 & 400 & 600 & 800 & 1000 & 1200 \\
\hline Step of mesh $h=\frac{1}{N}$ & $\frac{1}{100}$ & $\frac{1}{200}$ & $\frac{1}{300}$ & $\frac{1}{400}$ & $\frac{1}{600}$ & $\frac{1}{800}$ & $\frac{1}{1000}$ & $\frac{1}{1200}$ \\
\hline Number of triangles $n_{T}$ & 8341 & 34185 & 74581 & 135161 & 300275 & 531609 & 830985 & 1206771 \\
\hline Number of vertices $n_{S}$ & 4322 & 17394 & 37742 & 68182 & 151039 & 267006 & 416994 & 605187 \\
\hline
\end{tabular}

Table 2. Characteristics of the meshes tested

\begin{tabular}{|c|c|c|c|c|c|c|c|c|}
\hline Parameters $N$ & 1300 & 1400 & 1500 & 1600 & 1700 & 1800 & 1900 & 2000 \\
\hline Step of mesh $h=\frac{1}{N}$ & $\frac{1}{1300}$ & $\frac{1}{1400}$ & $\frac{1}{1500}$ & $\frac{1}{1600}$ & $\frac{1}{1700}$ & $\frac{1}{1800}$ & $\frac{1}{1900}$ & $\frac{1}{2000}$ \\
\hline Number of triangles $n_{T}$ & 1630175 & 1449489 & 1873567 & 2138511 & 2398679 & 2757041 & 3009721 & 3319791 \\
\hline Number of vertices $n_{S}$ & 817189 & 726696 & 939035 & 1071657 & 1201891 & 1381222 & 1507712 & 1662897 \\
\hline
\end{tabular}




\subsection{Finite element calculations of the coefficients of singularities}

The coefficients of discrete singularities $\left(\lambda_{i}^{h}\right)_{1 \leq i \leq 4}$ have been calculated by Free-fem++ 3.17 from the mixed variational formulation. 
Table 3. Finite element approximate calculations of the coefficients of singularities

\begin{tabular}{|c|c|c|c|c|}
\hline Step $h=\frac{1}{N}$ & $\lambda_{1}^{h}$ & $\lambda_{2}^{h}$ & $\lambda_{3}^{h}$ & $\lambda_{4}^{h}$ \\
\hline$N=100$ & $\lambda_{1}^{h}=0.0159255$ & $\lambda_{2}^{h}=0.00373141$ & $\lambda_{3}^{h}=0.00688557$ & $\lambda_{4}^{h}=-0.00019966$ \\
\hline$N=200$ & $\lambda_{1}^{h}=0.0159324$ & $\lambda_{2}^{h}=-0.000577502$ & $\lambda_{3}^{h}=0.0068856$ & $\lambda_{4}^{h}=-0.0002000520$ \\
\hline$N=300$ & $\lambda_{1}^{h}=0.0159337$ & $\lambda_{2}^{h}=0.00373333$ & $\lambda_{3}^{h}=0.00688911$ & $\lambda_{4}^{h}=-0.00020069$ \\
\hline$N=400$ & $\lambda_{1}^{h}=0.0159341$ & $\lambda_{2}^{h}=0.00373343$ & $\lambda_{3}^{h}=0.0068893$ & $\lambda_{4}^{h}=-0.000200074$ \\
\hline$N=600$ & $\lambda_{1}^{h}=0.0159344$ & $\lambda_{2}^{h}=0.00373351$ & $\lambda_{3}^{h}=0.00688944$ & $\lambda_{4}^{h}=-0.000200078$ \\
\hline$N=800$ & $\lambda_{1}^{h}=0.0159345$ & $\lambda_{2}^{h}=0.00373354$ & $\lambda_{3}^{h}=0.00688949$ & $\lambda_{4}^{h}=-0.00020008$ \\
\hline$N=1000$ & $\lambda_{1}^{h}=0.159346$ & $\lambda_{2}^{h}=0.00373355$ & $\lambda_{3}^{h}=0.00688951$ & $\lambda_{4}^{h}=-0.00020008$ \\
\hline$N=1200$ & $\lambda_{1}^{h}=0.0159346$ & $\lambda_{2}^{h}=0.00373355$ & $\lambda_{3}^{h}=0.00688953$ & $\lambda_{4}^{h}=-0.000200081$ \\
\hline
\end{tabular}


Table 4. Finite element approximate calculations of the coefficients of singularities

\begin{tabular}{|c|c|c|c|c|}
\hline Step $h=\frac{1}{N}$ & $\lambda_{1}^{h}$ & $\lambda_{2}^{h}$ & $\lambda_{3}^{h}$ & $\lambda_{4}^{h}$ \\
\hline$N=1300$ & $\lambda_{1}^{h}=0.0159346$ & $\lambda_{2}^{h}=-0.000577583$ & $\lambda_{3}^{h}=0.00688953$ & $\lambda_{4}^{h}=-0.000200081$ \\
\hline$N=1400$ & $\lambda_{1}^{h}=0.0159346$ & $\lambda_{2}^{h}=-0.000577583$ & $\lambda_{3}^{h}=0.00688953$ & $\lambda_{4}^{h}=-0.0002000081$ \\
\hline$N=1500$ & $\lambda_{1}^{h}=0.0159346$ & $\lambda_{2}^{h}=-0.000577584$ & $\lambda_{3}^{h}=0.00688954$ & $\lambda_{4}^{h}=-0.0002000081$ \\
\hline$N=1600$ & $\lambda_{1}^{h}=0.0159346$ & $\lambda_{2}^{h}=-0.000577584$ & $\lambda_{3}^{h}=0.00688954$ & $\lambda_{4}^{h}=-0.0002000081$ \\
\hline$N=1700$ & $\lambda_{1}^{h}=0.0159347$ & $\lambda_{2}^{h}=-0.000577584$ & $\lambda_{3}^{h}=0.00688954$ & $\lambda_{4}^{h}=-0.000200081$ \\
\hline$N=1800$ & $\lambda_{1}^{h}=0.0159347$ & $\lambda_{2}^{h}=-0.000577584$ & $\lambda_{3}^{h}=0.00688954$ & $\lambda_{4}^{h}=-0.000200081$ \\
\hline$N=1900$ & $\lambda_{1}^{h}=0.0161911$ & $\lambda_{2}^{h}=-0.000577584$ & $\lambda_{3}^{h}=0.00688954$ & $\lambda_{4}^{h}=-0.000200081$ \\
\hline$N=2000$ & $\lambda_{1}^{h}=0.0159347$ & $\lambda_{2}^{h}=-0.000577584$ & $\lambda_{3}^{h}=0.00688954$ & $\lambda_{4}^{h}=-0.000200081$ \\
\hline
\end{tabular}




\subsection{Direct calculation of the coefficients of singularities}

Singularities of the coefficients $\left(\lambda_{i}\right)_{1 \leq i \leq 4}$ were calculated directly by Free-fem ++3.17 using the integral formulas that provide the coefficients of singularities from singular functions and singular dual functions. 
Table 5. Direct calculation of the coefficients of singularities

\begin{tabular}{|c|c|c|c|c|}
\hline Step $h=\frac{1}{N}$ & $\lambda_{1}$ & $\lambda_{2}$ & $\lambda_{3}$ & $\lambda_{4}$ \\
\hline$N=100$ & $\lambda_{1}=0.0161818$ & $\lambda_{2}=0.00539393$ & $\lambda_{3}=0.00539393$ & $\lambda_{4}=-0.00107879$ \\
\hline$N=200$ & $\lambda_{1}=0.0161888$ & $\lambda_{2}=-0.00298689$ & $\lambda_{3}=0.00539627$ & $\lambda_{4}=-0.00107925$ \\
\hline$N=300$ & $\lambda_{1}=0.0161901$ & $\lambda_{2}=0.0053967$ & $\lambda_{3}=0.0053967$ & $\lambda_{4}=-0.00107934$ \\
\hline$N=400$ & $\lambda_{1}=0.0161906$ & $\lambda_{2}=0.00539686$ & $\lambda_{3}=0.00539686$ & $\lambda_{4}=-0.00107937$ \\
\hline$N=600$ & $\lambda_{1}=0.0161909$ & $\lambda_{2}=0.00539696$ & $\lambda_{3}=0.00539696$ & $\lambda_{4}=-0.00107939$ \\
\hline$N=800$ & $\lambda_{1}=0.0161910$ & $\lambda_{2}=0.005397$ & $\lambda_{3}=0.005397$ & $\lambda_{4}=-0.0010794$ \\
\hline$N=1000$ & $\lambda_{1}=0.0161911$ & $\lambda_{2}=0.00539702$ & $\lambda_{3}=0.00539702$ & $\lambda_{4}=-0.0010794$ \\
\hline$N=1200$ & $\lambda_{1}=0.0161911$ & $\lambda_{2}=0.00539703$ & $\lambda_{3}=0.00539703$ & $\lambda_{4}=-0.00107941$ \\
\hline
\end{tabular}


Table 6. Direct calculation of the coefficients of singularities

\begin{tabular}{|c|c|c|c|c|}
\hline Step $h=\frac{1}{N}$ & $\lambda_{1}$ & $\lambda_{2}$ & $\lambda_{3}$ & $\lambda_{4}$ \\
\hline$N=1300$ & $\lambda_{1}=0.0161911$ & $\lambda_{2}=0.00539703$ & $\lambda_{3}=0.00539703$ & $\lambda_{4}=-0.00107941$ \\
\hline$N=1400$ & $\lambda_{1}=0.0161911$ & $\lambda_{2}=0.00539703$ & $\lambda_{3}=0.00539703$ & $\lambda_{4}=-0.00107941$ \\
\hline$N=1500$ & $\lambda_{1}=0.0161911$ & $\lambda_{2}=0.00539704$ & $\lambda_{3}=0.00539704$ & $\lambda_{4}=-0.00107941$ \\
\hline$N=1600$ & $\lambda_{1}=0.0161911$ & $\lambda_{2}=0.00539704$ & $\lambda_{3}=0.00539704$ & $\lambda_{4}=-0.00107941$ \\
\hline$N=1700$ & $\lambda_{1}=0.0161911$ & $\lambda_{2}=0.00539704$ & $\lambda_{3}=0.00539704$ & $\lambda_{4}=-0.00107941$ \\
\hline$N=1800$ & $\lambda_{1}=0.0161911$ & $\lambda_{2}=0.00539704$ & $\lambda_{3}=0.00539704$ & $\lambda_{4}=-0.00107941$ \\
\hline$N=1900$ & $\lambda_{1}=0.0161911$ & $\lambda_{2}=0.00539704$ & $\lambda_{3}=0.00539704$ & $\lambda_{4}=-0.00107941$ \\
\hline$N=2000$ & $\lambda_{1}=0.0161911$ & $\lambda_{2}=0.00539704$ & $\lambda_{3}=0.00539704$ & $\lambda_{4}=-0.00107941$ \\
\hline
\end{tabular}


Table 7. Error estimates

\begin{tabular}{|c|c|c|c|c|}
\hline Step $h=\frac{1}{N}$ & $E_{1}^{h}$ & $E_{2}^{h}$ & $E_{3}^{h}$ & $E_{4}^{h}$ \\
\hline$N=100$ & $e_{1}=0.00012816$ & $e_{2}=0.000831259$ & $e_{3}=0.000745819$ & $e_{4}=0.00043941$ \\
\hline$N=200$ & $e_{1}=0.000128215$ & $e_{2}=0.00298689$ & $e_{3}=0.000746143$ & $e_{4}=0.000439601$ \\
\hline$N=300$ & $e_{1}=0.000128226$ & $e_{2}=0.000831687$ & $e_{3}=0.000746203$ & $e_{4}=0.000439636$ \\
\hline$N=400$ & $e_{1}=0.000128229$ & $e_{2}=0.000831711$ & $e_{3}=0.000746224$ & $e_{4}=0.000439648$ \\
\hline$N=600$ & $e_{1}=0.000128232$ & $e_{2}=0.000831727$ & $e_{3}=0.000746239$ & $e_{4}=0.000439657$ \\
\hline$N=800$ & $e_{1}=0.000128233$ & $e_{2}=0.000831733$ & $e_{3}=0.000746245$ & $e_{4}=0.00043966$ \\
\hline$N=1000$ & $e_{1}=0.000128233$ & $e_{2}=0.000831736$ & $e_{3}=0.000746247$ & $e_{4}=0.000439662$ \\
\hline$N=1200$ & $e_{1}=0.000128233$ & $e_{2}=0.000831737$ & $e_{3}=0.000746248$ & $e_{4}=0.000439663$ \\
\hline
\end{tabular}




\subsection{Error estimates}

For each step of the discretization, the errors are in the order of $O\left(h^{2}\right)$ in $L^{2}(\Omega)$ determined by:

$$
E_{i}^{h}=\left\|\lambda_{i}-\lambda_{i}^{h}\right\|_{L^{2}(\Omega)} \text { for all } i \in\{1,2,3,4\} .
$$


Table 8. Error estimates

\begin{tabular}{|c|c|c|c|c|}
\hline Step $h=\frac{1}{N}$ & $E_{1}^{h}$ & $E_{2}^{h}$ & $E_{3}^{h}$ & $E_{4}^{h}$ \\
\hline$N=1300$ & $e_{1}=0.000128234$ & $e_{2}=-0.000298731$ & $e_{3}=0.000746249$ & $e_{4}=0.000439663$ \\
\hline$N=1400$ & $e_{1}=0.000128234$ & $e_{2}=-0.000298731$ & $e_{3}=0.000746249$ & $e_{4}=0.000439663$ \\
\hline$N=1500$ & $e_{1}=0.000128234$ & $e_{2}=0.000298731$ & $e_{3}=0.000746249$ & $e_{4}=0.000439663$ \\
\hline$N=1600$ & $e_{1}=0.000128234$ & $e_{2}=0.00298731$ & $e_{3}=0.00074625$ & $e_{4}=0.000439663$ \\
\hline$N=1700$ & $e_{1}=0.000128234$ & $e_{2}=0.00298731$ & $e_{3}=0.00074625$ & $e_{4}=0.000439663$ \\
\hline$N=1800$ & $e_{1}=0.000128234$ & $e_{2}=0.00298731$ & $e_{3}=0.00074625$ & $e_{4}=0.000439664$ \\
\hline$N=1900$ & $e_{1}=0.000128234$ & $e_{2}=0.00298731$ & $e_{3}=0.00074625$ & $e_{4}=0.000439664$ \\
\hline$N=2000$ & $e_{1}=0.000128234$ & $e_{2}=0.00298731$ & $e_{3}=0.00074625$ & $e_{4}=0.000439664$ \\
\hline
\end{tabular}




\subsection{Representative curves}

The curves below were drawn through the Scilab free software of INRIA.

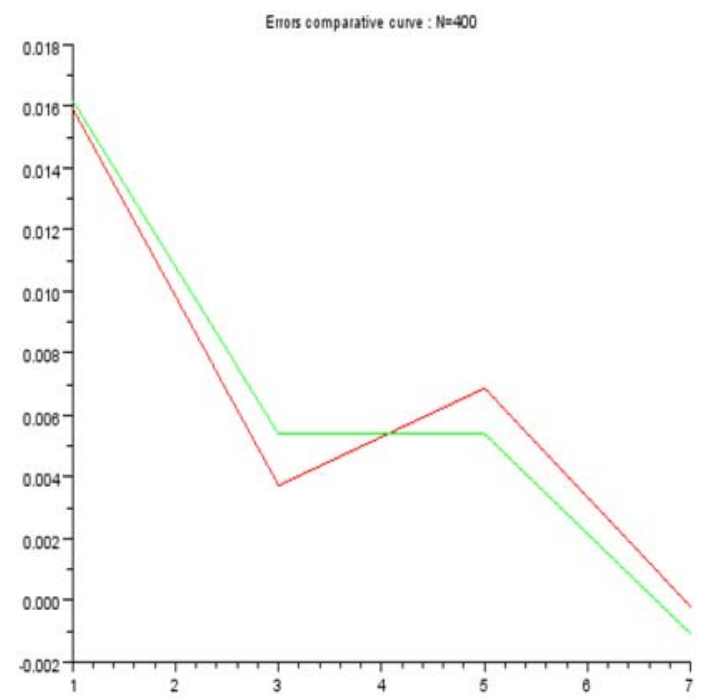

Figure 2. Coefficients of singularities: $\frac{1}{400}$.

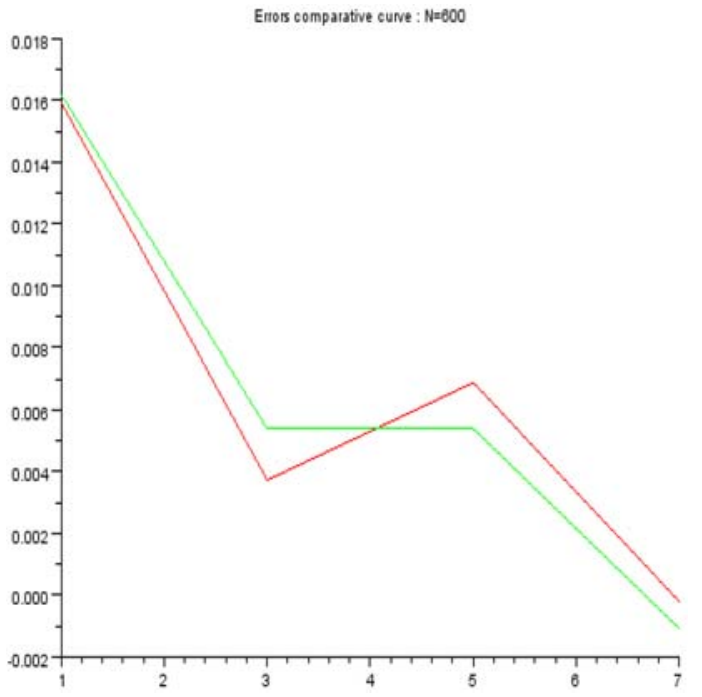

Figure 3. Coefficients of singularities: $\frac{1}{600}$. 


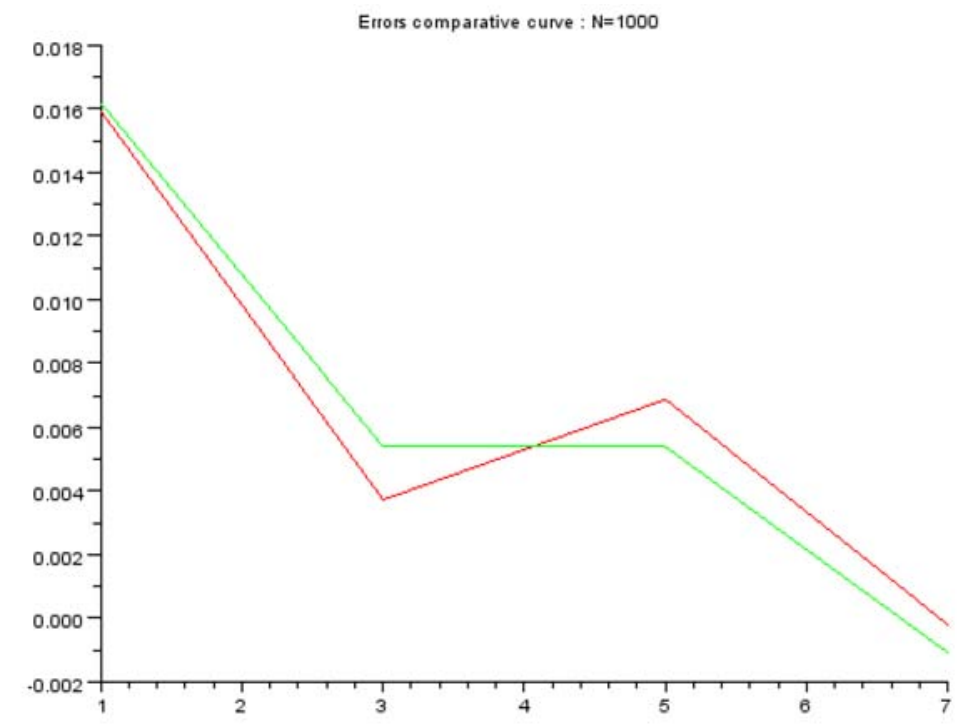

Figure 4. Coefficients of singularities: $\frac{1}{1000}$.

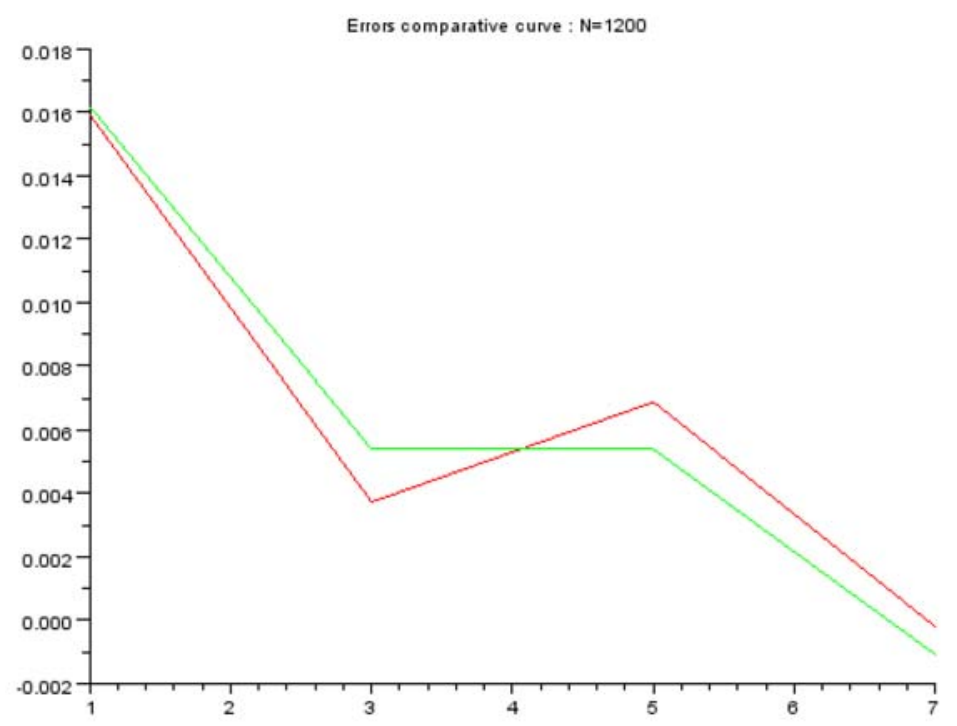

Figure 5. Coefficients of singularities: $\frac{1}{1200}$. 


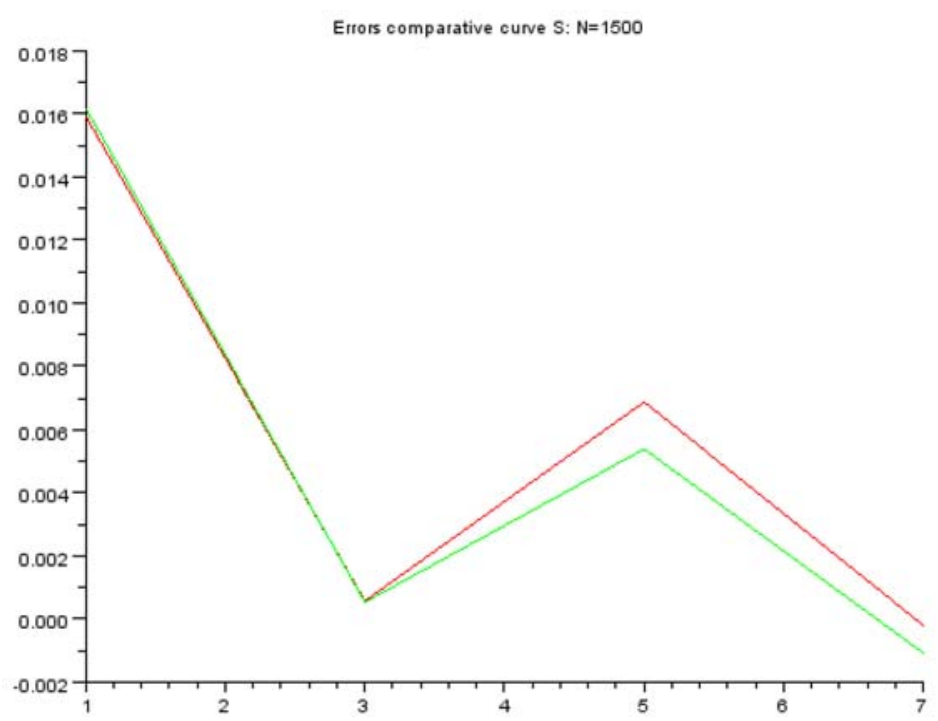

Figure 6. Coefficients of singularities: $\frac{1}{1500}$.

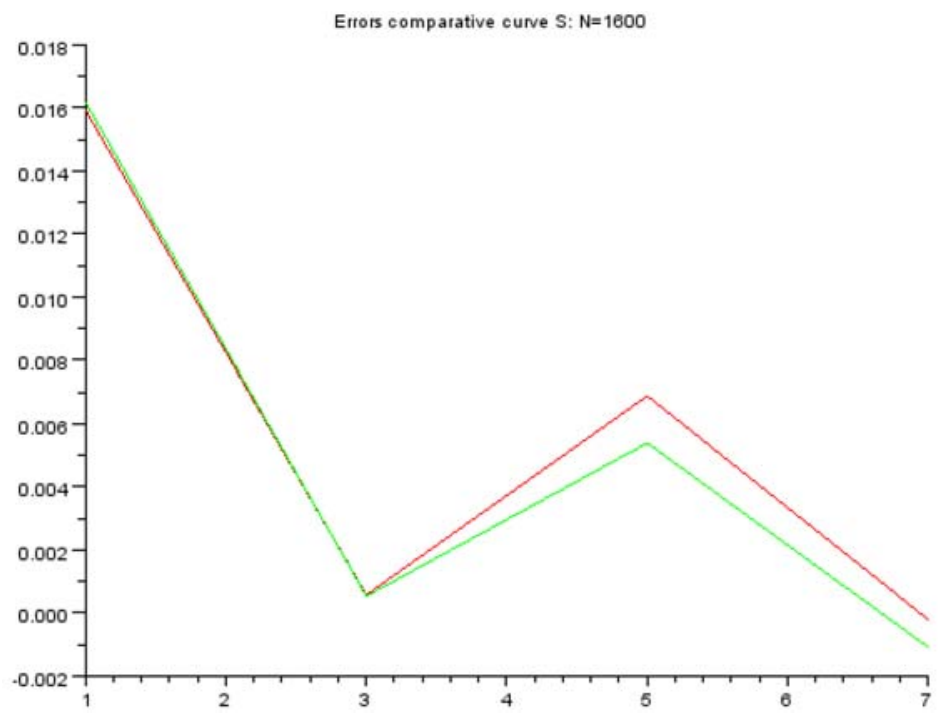

Figure 7. Coefficients of singularities: $\frac{1}{1600}$. 


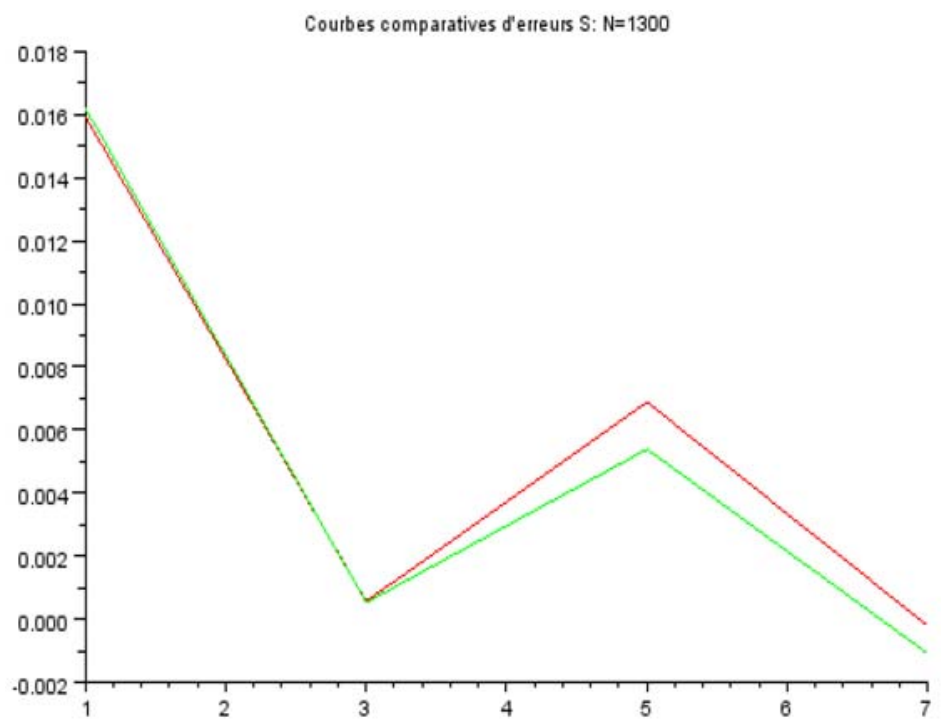

Figure 8. Coefficients of singularities: $\frac{1}{1300}$.

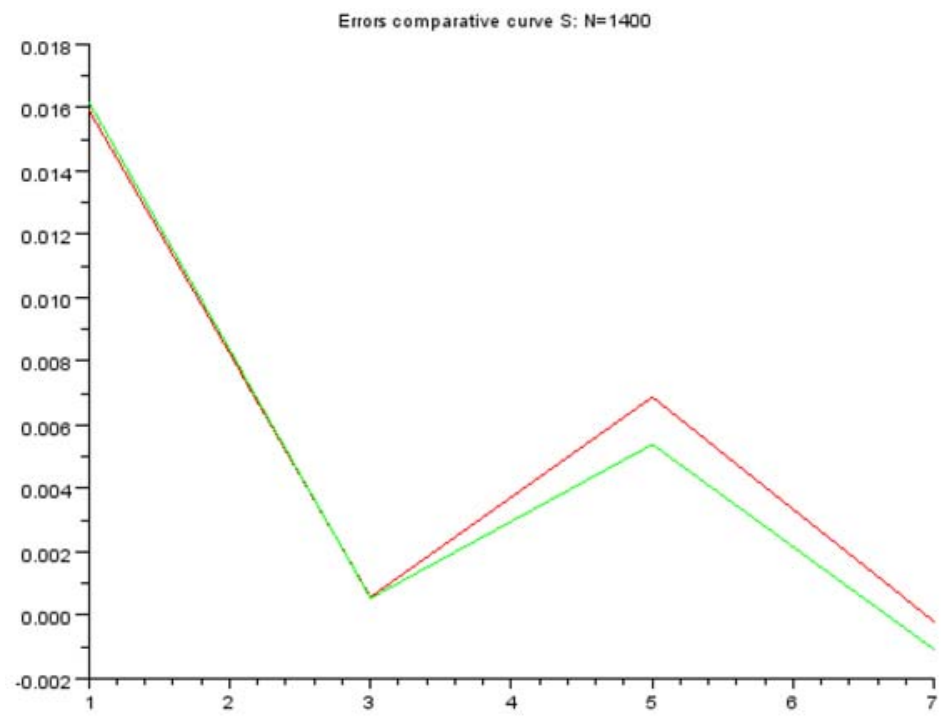

Figure 9. Coefficients of singularities: $\frac{1}{1400}$. 


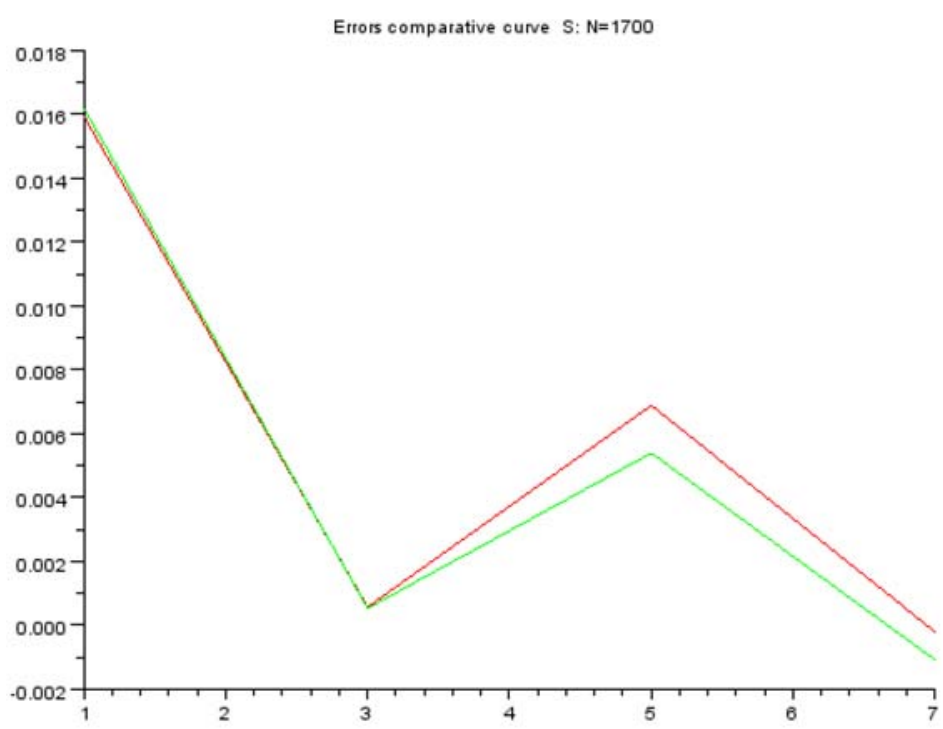

Figure 10. Coefficients of singularities: $\frac{1}{1700}$.

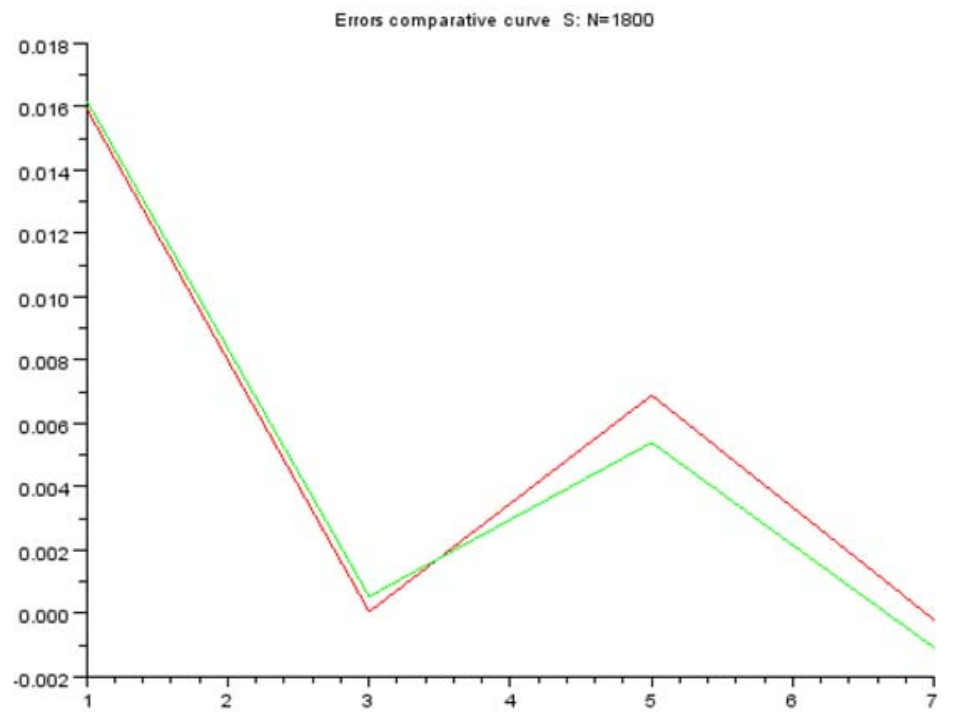

Figure 11. Coefficients of singularities: $\frac{1}{1800}$. 


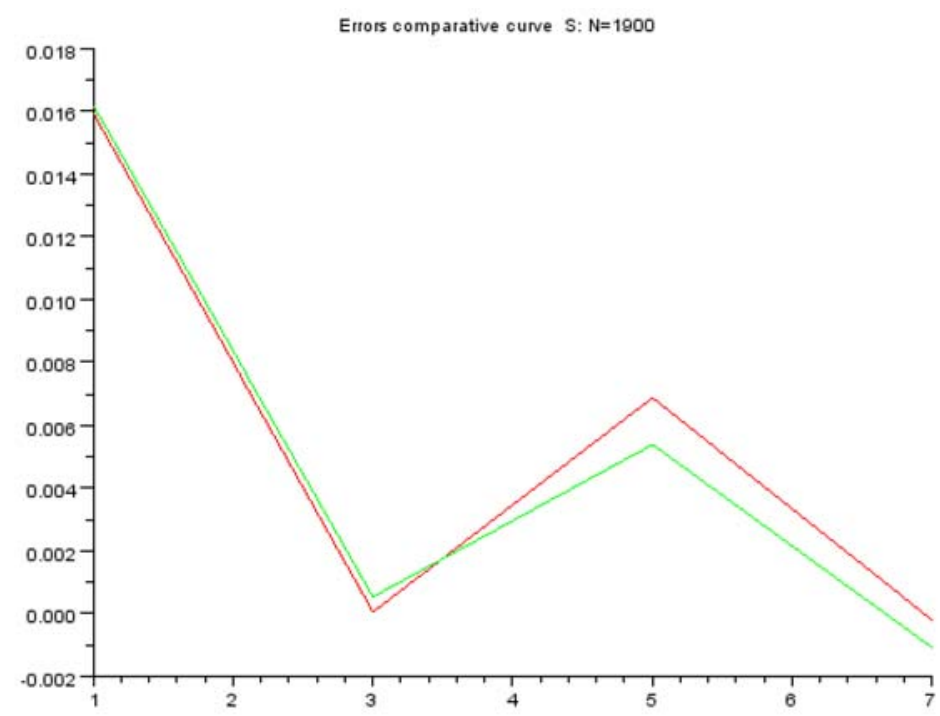

Figure 12. Coefficients of singularities: $\frac{1}{1900}$.

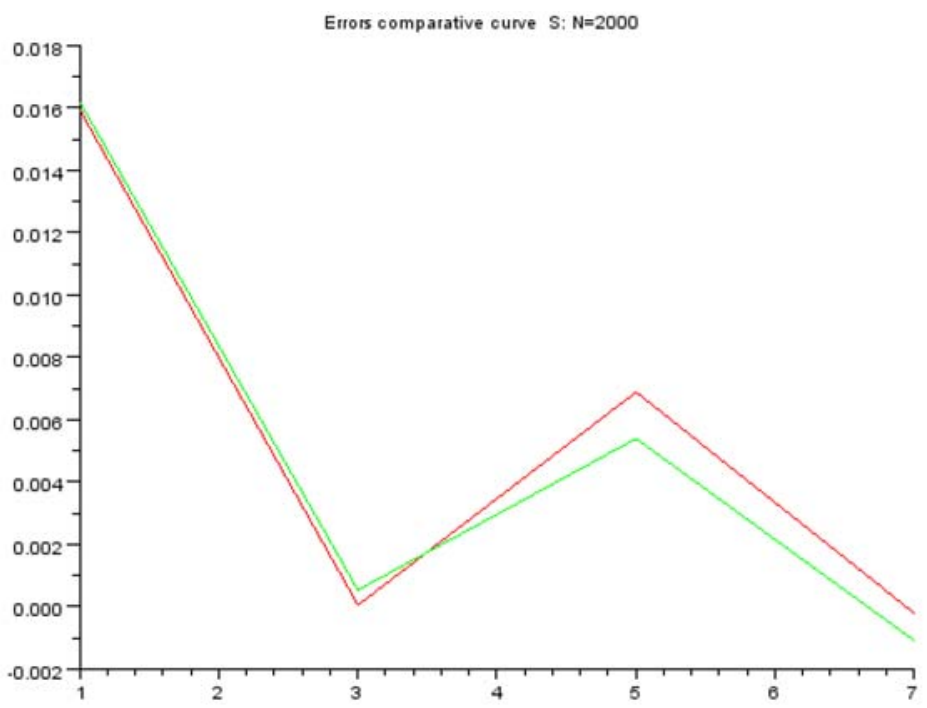

Figure 13. Coefficients of singularities: $\frac{1}{2000}$. 


\section{Interpretation of Numerical Tests}

(1) These numerical results made us realize that the respective values of the coefficients of singularities are very close to the coefficients of singularities $\lambda_{1}, \lambda_{2}, \lambda_{3}$. However, the value of $\lambda_{4}$ is negative (relatively negligible) compared to other coefficients of singularities (on algebric value).

(2) Representations of the curves of singularity coefficients obtained from direct calculations (green) and those obtained from mixed finite elements (in red) are nearly the same and if the mesh becomes very small, amplitude between the two curves becomes very small, narrows and stabilizes when the mesh becomes more refined. Hence the effects of convergence in all cases remain.

(3) Knowing that the direct calculation of the coefficients of singularities is not always possible, and since the maximum amplitude error of approximation is relatively small, we can approximate them properly by the coefficients of singularities obtained by mixed finite elements, that can be calculated in general with Free-fem++ to estimate the crack propagation.

In the final analysis, the calculation methods of singular solutions and coefficients of singularities presented here uses finite element affine type $P 1$. Besides the result sets of convergence can improve stabilities obtained using finite element $P 2$ and $P 3$ with Free-fem++.

Therefore, we obtain a priori error estimates more interesting and so asymptotically greater convergence on the coefficients of crack propagation in the field. More refined mesh is better the convergence and stability of the singularities obtained. However, the cost of the numerical implementation is very high and the grid spacing exceeds 1600, the coefficients of singularities stabilize up to 8 decimal relative. 


\section{Acknowledgement}

The paper thanks for the Dean of our faculty FASTEF of UCAD and CEA-MITIC which allowed me to pay the expenses of the publication.

\section{References}

[1] M. Amara, A. Chatti and F. El. Dabaghi, Elément fini optimal de classe $C^{0}$ pour des problèmes de fissures planes, R. R. INRIA, 1998.

[2] M. Amara and M. Moussaoui, Approximation de coefficients de singularités, C. R. Acad. Sci. Paris Sér. I Math. 313(5) (1991), 335-338.

[3] G. Bayili, C. Seck, A. Séne and M. T. Niane, Control and cancellation singularities of Bilaplacian in a cracked domains, Journal of Mathematics Research 4(4) (2012), 35-39.

$$
\text { DOI: http://dx.doi.org/10.5539/jmr.v4n4p35 }
$$

[4] P. G. Ciarlet and P. A. Raviart, A mixed finite element method for the biharmonic equation, Mathematical Aspect of Finite Elements in Partial Differential Equations, Carl de Boor Ed, Academic Press, New York (1974), 125-145.

[5] P. Grisvard, Problème de dirichlet dans un domaine non régulier, C. R. Acad. Sci. Paris Sér. A 278 (1974), 1615-1617.

[6] P. Grisvard, Elliptic Problems in Nonsmooth Domains, Volume 24 of Monographs and Studies in Mathematics Pitman (Advanced Publishing Program), Boston, MA, 1985.

[7] P. Grisvard, Contrôlabilité exacte des solutions de l'équation des ondes en présence de singularités, Journal de Mathématiques Pures et Appliquées 68(12) (1989), 215-259.

[8] V. A. Kondrat'ev, Boundary value problems for elliptic equations in domains with conical or angular points, Tr. Mosk. Mat. Obs. 16 (1967), 209-292.

[9] M. Moussaoui, Singularités des solutions du problème mêlé, contrôlabilité exacte et stabilisation frontière, In Élasticité Viscoélasticité et Contrôle Optimal (Lyon, 1995), volume 2 of ESAIM Proc., pages 195-201 (electronic). Soc. Math. Appl. Indust. Paris 1997.

[10] M. T. Niane, G. Bayili, A. Sène, A. Sène and M. Sy, Is it possible to cancel singularities in a domain with corners and cracks? C. R. Math. Acad. Sci. Paris 343(2) (2006), 115-118.

[11] H. Timouyas, Analyse et Analyse Numérique des Singularités en Électromagnétisme, Thèse Unique, Ecole Centrale de Lyon, France, 2003. 